\title{
Clinical significance of CSF3R, SRSF2 and SETBP1 mutations in chronic neutrophilic leukemia and chronic myelomonocytic leukemia
}

\author{
Yuan Ouyang ${ }^{1,2, *}$, Chun Qiao ${ }^{2, *}$, Yu Chen ${ }^{1}$, Su-Jiang Zhang ${ }^{1,2}$ \\ ${ }^{1}$ Department of Hematology, Ruijin Hospital North Shanghai Jiao Tong University School of Medicine, Shanghai 200000, \\ China \\ ${ }^{2}$ Department of Hematology, The First Affiliated Hospital of Nanjing Medical University, Jiangsu Province Hospital, Collaborative \\ Innovation Center For Cancer Personalized Medicine, Nanjing Medical University, Nanjing 210029, China \\ *These authors contributed equally to this work
}

Correspondence to: Su-Jiang Zhang, email: zbruce.zhang@gmail.com

Keywords: CNL, CMML, gene mutation, CSF3R, SRSF2

Received: December 29, 2015

Accepted: January 27, 2017

Published: February 15, 2017

\section{ABSTRACT}

Chronic neutrophilic leukemia (CNL) and chronic myelomonocytic leukemia (CMML) are rare hematologic neoplasms. We performed CSF3R, SRSF2 and SETBP1 mutational analyses in $10 \mathrm{CNL}$ and $56 \mathrm{CMML}$ patients. In this sample cohort, $80 \%$ of CNL patients harbored CSF3R mutations, of which the CSF3R T618I mutation was dominant. Mutations in CSF3R and SETBP1 were found in $7.1 \%$ and $5.3 \%$ CMML patients respectively, while $25 \%$ of CMML patients carried SRSF2 mutations. Strikingly, we identified that all of the CSF3R mutations detected in CMML patients were represented by a P733T mutation. The CSF3R P733T mutation represents a novel CSF3R mutation. In addition, none of the four CSF3R P733T mutated patients carried SRSF2 mutations [0/14 (0\%) patients with combined CSF3R P733T and SRSF2 mutations vs. 4/42 (9.5\%) with CSF3R P733T and wt SRSF2, $P<0.001]$. Both mut SRSF2 and mut SETBP1 patients had shorter overall survival (OS) and progression-free survival (PFS) compared to patients with wt SRSF2 $(P<0.001$ both) and wt SETBP1 ( $P<0.001$ and $P=0.02$, respectively). While we found no significant differences in OS and PFS as a consequence of CSF3R mutation status, our work suggest that the CSF3R T618I mutation is a diagnostic marker with good specificity and sensitivity for CNL. In conclusion, our study highlights effective diagnostic and prognostic markers of CNL and CMML patients in the Chinese population.

\section{INTRODUCTION}

CNL is a rare hematologic neoplasm that is diagnosed largely based on exclusion of underlying causes of reactive neutrophilia and/or the lack of specific molecular markers of other hematological malignancies [1]. Until the recent discoveries of CSF3R and SETBPI mutations [2], no recurrent genetic abnormalities have been identified in CNL.

CSF3R (G-CSF-R), the colony-stimulating factor 3 receptor, is a trans-membrane protein which plays a prominent role in the growth and differentiation of granulocytes [3]. While CSF3R mutations are most commonly found in severe congenital neutropenia (SCN), the rates of CSF $3 R$ mutations rises sharply upon progression to secondary acute myeloid leukemia (sAML) [4-6]. Thus,
CSF3R mutations may critically influence on disease progression to AML, although the types of truncation mutations that associate with SAML are rarely detected in other disorders, including de novo AML6. Recently, acquired $C S F 3 R$ mutations (in particular the CSF3R T618I mutation) were described in a majority of patients diagnosed with CNL or atypical chronic myeloid leukemia (aCML) $[2,7]$. While the relevance of CSF3R mutations in CMML has also been investigated, discrepancies exist among different studies. For example, Kosmider et al. identified about 3\% of CMML patients with CSF3R somatic mutations, while Pardanani et al failed to identify CSF3R mutations in CMML $[8,9]$.

CMML is the most frequent entity among myeloproliferative/myelodysplastic neoplasms (MDS/ MPN) [10]. About $90 \%$ of CMML patients carry genomic 
aberrations, which includes mutations in genes encoding for epigenetic regulators (TET2, ASXL1, DNMT3A, EZH2, IDH1, IDH2), spliceosome components (SRSF2, SF3B1, ZRSF2, U2AF1), transcription factors (RUNX1, NPM1, TP53) and other signaling molecules (NRAS, KRAS, CBL, JAK2, FLT3) [11]. Of the many genes found mutated in CMML, SRSF2 mutations are dominating. SRSF2 mutations may excert oncogenic activity by regulating alternative splicing through prevention of exon skipping [12]. The mutational frequency of $S R S F 2$ in MDS, CMML and SAML were reported to be 10-15\%, $21-47 \%$ and $6.5-24 \%$, respectively [13]. Interestingly, an $S R S F 2$ mutation was also detected in one CNL patient [14].

Apart from mutations in $C S F 3 R$ and $S R S F 2$, mutations in SETBP1 has also been demonstrated in hematological malignancies. Piazza et al. discovered SETBP 1 mutations in $24 \%$ of aCML, $10 \%$ of unclassified MDS/MPN, $4 \%$ of CMML and in $25 \%$ of CNL cases [15]. Likewise, Damm et al. observed a frequency of SETBP1 mutations of $1.7 \%, 2.2 \%$ and $6.2 \%$ in SAML, MDS and CMML, respectively [16].

Here, we have unveiled the frequency, clinical significance and prognostic relevance of mutations in CSF3R, SETBP1 and SRSF 2 in a cohort of 10 CNL and 56 CMML patients, with the aim of providing insights into the development of effective diagnostic and prognostic tools of CNL and CMML patients in the Chinese population.

\section{RESULTS}

\section{Mutational landscape in the patients}

In our study, we found that $80 \%(8 / 10)$ of CNL patients harbored $C S F 3 R$ mutations. Intriguingly, $87.5 \%$ (7/8) of patients carried CSF3R T618I substitution mutations and 2 carried double mutations (T618I together with W818X and Q749X, respectively). One CNL patient carried a $C S F 3 R$ P733T mutation (Figure 1A). The patient with T618I/Q749X double mutations also had a SETBP1 D874N mutation. In 56 CMML patients, 25\% (14/56) of patients were found to have SRSF2 mutations (P95H (11 patients), P95L (1 patient), P95R (1 patient) and P95fs*19 (1 patient)) (Figure 1B). In addition, 7.1\% (4/56) of CMML patients had a $C S F 3 R$ P733T mutation and 5.3\% (3/56) had SETBP1 mutations (I871T (2 patients) and D868N (1 patients)) (Figure 2). No CSF3R, SRSF2 or SETBP1 mutations were identified in patients diagnosed with MDS, CEL or in healthy donors (Table 1). All of the gene mutations identified in our study were somatic mutations.

\section{Impact of mutation status on clinical and biological characteristics}

CSF3R mutated CNL patients constituted of 7 males and 1 female. The median age was 39 (range 27 to 92 years $)$. These patients had $(45 \pm 38.39) \times 10^{9} / \mathrm{L}$ of mean white blood cell (WBC) count, $(40.73 \pm 37.41) \times 10^{9} / \mathrm{L}$ of mean neutrophil count, $(87.7 \pm 5.53) \%$ of mean neutrophil percentage, $(100.25 \pm 33.1) \mathrm{g} / \mathrm{L}$ of mean hemoglobin(HB) level and $(148.13 \pm 124.34) \times 10^{9} / \mathrm{L}$ of mean blood platelet cell (BPC) count. Further, blast cell count of peripheral blood $(\mathrm{PB})$ and bone marrow $(\mathrm{BM})$ were $(2.13 \pm 4.02) \%$ and $(3.63 \pm 2.62) \%$, respectively. One patient with an abnormal karyotype $(47, \mathrm{XY},+8)$ was observed. During a median follow-up of 22 months (range: 4-37 months), 2 CSF3R mutated CNL patients died (one case died because of disease evolution into ANLL-M2). Of two wt CSF3R CNL patients, an evolution to acute plasma cell leukemia (APCL) was observed in the patient with MGUS-CNL (Table 2). In CMML, none of the $4 C S F 3 R$ mutated patients were detected with $S R S F 2$ mutations [0/14(0\%) vs. $4 / 42(9.5 \%), P<0.001]$; 1 of the 3 patients with SETBP1 mutations also had SRSF2 mutations [1/14(7.1\%) vs. $2 / 42(4.8 \%), P>0.05]$. Characteristics such as age, gender, WHO category, FAB category, karyotype, blood cell counts and CPSS risk stratification did not reveal any difference between mutated SRSF2 patients and wt SRSF2 patients $(P>0.05)$ (Table 3$)$.

Patients with CSF3R mutation had higher HB levels $(P=0.05)$ compared to wt $C S F 3 R$ patients. However, no statistically significant difference was identified between mut $C S F 3 R$ and wt $C S F 3 R$ CMML patients in terms of age, gender, WHO category, FAB category, karyotype, other blood cell counts and CPSS risk stratification. In terms of treatment for the investigated patients, we normally used hydroxyurea and Interferon- $\alpha$ for CNL patients, and demethylation therapy, combined chemotherapy, hydroxyurea, immunomodulation therapy and supportive treatment for CMML patients.

\section{SRSF2 and SETBP1 mutations are independent predictors of poor survival for CMML patients}

We next focused our prognostic analysis on the impact of mutation status in CMML patients. Overall, during a median follow-up period of 20 months (range: 2-60 months), 14(25\%) patients died, mainly due to progression to AML(10/14). Both SRSF2 and SETBP1 mutated patients showed shorter progression-free survival (PFS) and overall survival (OS) compared with wt SRSF2 (both $P<0.001$ ) and wt SETBP1 ( $P=0.02$ and $P<0.001)$ patients. In addition, we observed that patients with CSF3R mutations had longer PFS and OS compared to wt $C S F 3 R$ patients. However, the difference was not statistically significant $(P>0.05)$ (Figure 3$)$.

In univariate analysis, SRSF2 mutations (HR, 4.74; $95 \% \mathrm{CI}, 1.811$ to $12.42 ; P=0.002$ for $\mathrm{OS}$; HR, 15.39; $95 \%$ CI, 3.25 to $72.81 ; P=0.001$ for PFS, respectively), SETBP1 mutations (HR, 8.25; 95\% CI, 2.12 to 32.07; $P=0.002$ for OS; HR, 5.29; 95\% CI, 1.10 to 25.51; $P=0.038$ for $\mathrm{PFS}$, respectively), higher BM blast counts (HR, $1.18 ; 95 \% \mathrm{CI}, 1.05$ to $1.32 ; P=0.006$ for 
Table 1: CSF3R, SETBP1 and SRSF2 mutational status in different hematological malignancies

\begin{tabular}{|l|c|c|c|}
\hline \multicolumn{1}{c|}{ Diagnosis } & CSF3R Mutation (\%) & $1 / 10(10)$ & SRSF2 Mutation (\%) \\
\hline CNL & $8 / 10(80)$ & $3 / 56(5.3)$ & $0 / 10$ \\
\hline CMML & $4 / 56(7.1)$ & $0 / 10$ & $14 / 56(25)$ \\
\hline CEL & $0 / 10$ & $0 / 20$ & $0 / 10$ \\
\hline MDS & $0 / 20$ & $0 / 20$ \\
\hline
\end{tabular}

CNL: chronic neutrophilic leukemia; CMML: chronic myelomonocytic leukemia; CEL: chronic eosinophilic leukemia; MDS: myelodysplastic syndromes.

Table 2: Clinical characteristics and laboratory variables of CNL patients

\begin{tabular}{|c|c|c|c|c|c|c|c|c|c|c|c|c|c|c|c|}
\hline No. & Age & Sex & Diagnosis & WBC & N\% & HB & BPC & $\begin{array}{l}\text { PB } \\
\text { Blast }\end{array}$ & $\begin{array}{l}\text { BM } \\
\text { Blast }\end{array}$ & Karyotypes & CSF3R & SETBP1 & $S R S F 2$ & $\begin{array}{l}\text { Disease } \\
\text { progression }\end{array}$ & Death \\
\hline 1 & 33 & $\mathrm{M}$ & $\mathrm{CNL}$ & 21.6 & 94.1 & 153 & 115 & 0 & 1.2 & NK & $\begin{array}{c}\text { T618I/ } \\
\text { W818X }\end{array}$ & WT & WT & $\mathrm{N}$ & $\mathrm{N}$ \\
\hline 2 & 65 & $\mathrm{M}$ & $\mathrm{CNL}$ & 26.9 & 86.0 & 76 & 17 & 0 & 3.6 & NK & $\begin{array}{l}\text { T618I/ } \\
\text { Q749X }\end{array}$ & D874N & WT & $\mathrm{N}$ & $\mathrm{N}$ \\
\hline 3 & 27 & F & $\mathrm{CNL}$ & 62.5 & 80.3 & 72 & 17 & 10 & 6.4 & NK & T618I & WT & WT & Y & Y \\
\hline 4 & 56 & M & $\mathrm{CNL}$ & 134.5 & 96.1 & 118 & 20 & 7 & 8.4 & NK & T618I & WT & WT & $\mathrm{N}$ & $\mathrm{N}$ \\
\hline 5 & 34 & M & $\mathrm{CNL}$ & 26.5 & 81.2 & 74 & 224 & 0 & 3.6 & $\begin{array}{c}47, \mathrm{XY} \\
+8\end{array}$ & T618I & WT & WT & $\mathrm{N}$ & $\mathrm{N}$ \\
\hline 6 & 32 & M & $\mathrm{CNL}$ & 28.0 & 86.8 & 63 & 188 & 0 & 3.6 & NK & T618I & WT & WT & $\mathrm{N}$ & $\mathrm{N}$ \\
\hline 7 & 44 & M & $\mathrm{CNL}$ & 39.7 & 88.6 & 129 & 273 & 0 & 1.6 & NK & T618I & WT & WT & $\mathrm{N}$ & $\mathrm{Y}$ \\
\hline 8 & 92 & M & $\mathrm{CNL}$ & 20.6 & 88.5 & 117 & 331 & 0 & 0.0 & NK & P733T & WT & WT & $\mathrm{N}$ & $\mathrm{N}$ \\
\hline 9 & 57 & M & $\mathrm{CNL}$ & 84.8 & 96.1 & 81 & 50 & 0 & 1.6 & NK & WT & WT & WT & $\mathrm{N}$ & $\mathrm{N}$ \\
\hline 10 & 77 & M & $\begin{array}{l}\text { MGUS- } \\
\text { CNL }\end{array}$ & 55.2 & 90.2 & 129 & 227 & 0 & 0.0 & NK & WT & WT & WT & $\mathrm{Y}$ & Y \\
\hline
\end{tabular}

CNL, Chronic neutrophilic leukemia; MGUS-CNL, undetermined significance monoclonal gammopathy associated with chronic neutrophilic leukemia; WBC, white blood cell; N\%, neutrophil percentage; HB, hemoglobin; BPC, blood platelet cell; PB Blast, blast cell count of peripheral blood; BM Blast, blast cell count of bone marrow; NK, abnormal karyotype; WT, wild type; CSF3R, colony-stimulating factor 3 receptor; SETBP1, SET-binding protein 1; $S R S F 2$, serine/arginine-rich splicing factor 2.

OS; HR, $1.14 ; 95 \%$ CI, 1.01 to $1.29 ; P=0.029$ for PFS, respectively), higher PB blast counts (HR, $1.14 ; 95 \% \mathrm{CI}$, 1.02 to $1.27 ; P=0.02$ for OS; HR, $1.13 ; 95 \% \mathrm{CI}, 1.01$ to $1.26 ; P=0.028$ for PFS, respectively), higher peripheral blood mononuclear cells (PBMC) (HR, 1.13; 95\% CI,

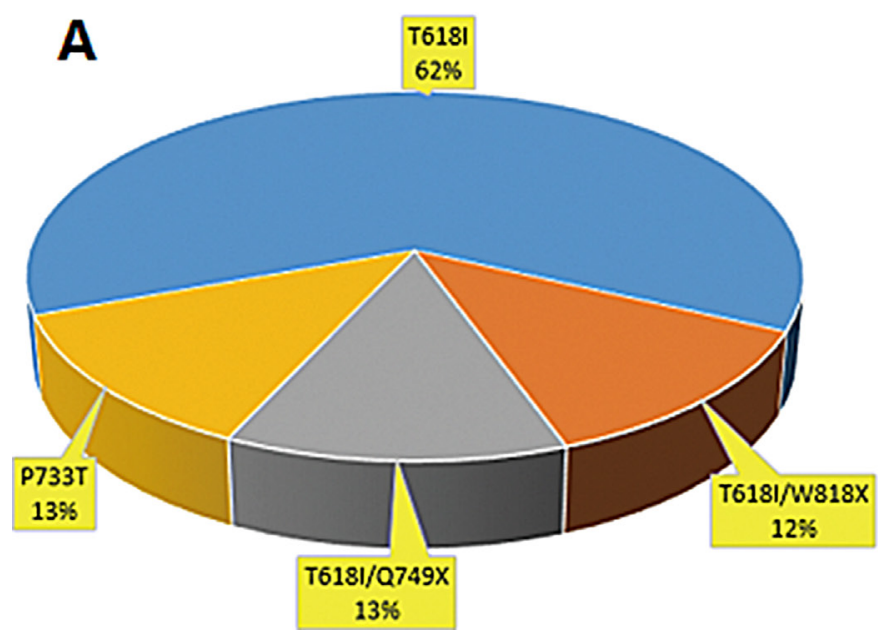

1.02 to $1.25 ; P=0.015$ for OS) and older age (HR, 1.04; $95 \% \mathrm{CI}, 1.01$ to $1.07 ; P=0.017$ for $\mathrm{OS}$ ) suggested a poor prognosis, while HB (HR, $0.941 ; 95 \%$ CI, 0.941 to 0.998 ; $P=0.038$ for PFS) was associated with a favorable PFS. Overall, there was no statistically significant difference
B

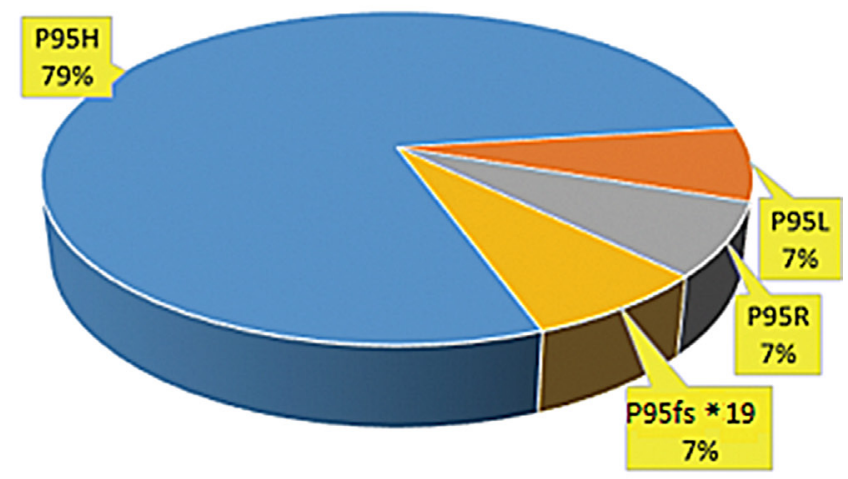

Figure 1: Frequency distribution of CSF3R and SRSF2 mutations in CNL and CMML patients. (A) In 10 patients with CNL, 8(8/10, 80\%) patients had CSF3R mutation and 7(7/8, 87.5\%) of them were with CSF3R T618I. (B) In 56 CMML patients, 14(14/56, $25 \%$ ) patients were found to have SRSF2 mutations, including P95H, P95L, P95R and P95fs*19. 
Table 3: CMML: patient characteristics and correlation with SRSF2 mutation status

\begin{tabular}{|c|c|c|c|}
\hline variables & $\operatorname{SRSF2} \operatorname{MUT}(n=14)$ & SRSF2 WT $(n=42)$ & $P$ \\
\hline Sex (female/male) & $4 / 10$ & $15 / 27$ & $>0.05$ \\
\hline Age (years) & $66.7(25-82)$ & $62.4(36-94)$ & $>0.05$ \\
\hline $\begin{array}{l}\text { WHO subtypes } \\
\text { CMML-1 } \\
\text { CMML-2 }\end{array}$ & $\begin{array}{c}10 \\
4\end{array}$ & $\begin{array}{c}33 \\
9\end{array}$ & $>0.05$ \\
\hline $\begin{array}{l}\text { FAB subtypes } \\
\text { CMML-MD } \\
\text { CMML-MP }\end{array}$ & $\begin{array}{c}2 \\
12\end{array}$ & $\begin{array}{l}10 \\
32\end{array}$ & $>0.05$ \\
\hline $\begin{array}{l}\text { CPSS } \\
\text { low risk } \\
\text { intermediate-1 risk } \\
\text { intermediate- } 2 \text { risk } \\
\text { high risk }\end{array}$ & $\begin{array}{l}2 \\
7 \\
5 \\
0\end{array}$ & $\begin{array}{c}4 \\
30 \\
7 \\
1\end{array}$ & $>0.05$ \\
\hline BM Blast (\%) & $3.13 \pm 1.7$ & $3.04 \pm 1.8$ & $>0.05$ \\
\hline PB Blast $(\%)$ & $3.13 \pm 1.7$ & $3.18 \pm 1.87$ & $>0.05$ \\
\hline WBC $\left(\times 10^{9} / \mathrm{L}\right)$ & $36.1 \pm 17.9$ & $33.0 \pm 13.1$ & $>0.05$ \\
\hline $\mathrm{HB}(\mathrm{g} / \mathrm{L})$ & $98.4 \pm 16.7$ & $94.4 \pm 11.5$ & $>0.05$ \\
\hline $\mathrm{BPC}\left(\times 10^{9} / \mathrm{L}\right)$ & $225.6 \pm 116.3$ & $222.4 \pm 135.8$ & $>0.05$ \\
\hline Mono $\left(\times 10^{9} / \mathrm{L}\right)$ & $4.58 \pm 3.1$ & $3.2 \pm 1.08$ & $>0.05$ \\
\hline Mono (\%) & $12.8 \pm 3.38$ & $15.1 \pm 4.82$ & $>0.05$ \\
\hline CSF3R mutation & $0 / 10$ & $4 / 42$ & $<0.001$ \\
\hline SETBP1 mutation & $2 / 14$ & $1 / 42$ & $>0.05$ \\
\hline
\end{tabular}

CMML, chronic myelomonocytic leukemia; WHO, World Health Organization; FAB, French, American and Britain; CMMLMD, CMML-myelodysplastic; CMML-MD, CMML- myeloproliferative; MUT, mutated; WT, wild type; CPSS, CMML Prognostic Scoring System; PB Blast, blast cell count of peripheral blood; BM Blast, blast cell count of bone marrow; WBC, white blood cell; HB, hemoglobin; BPC, blood platelet cell; Mono, monocyte.

in OS and PFS based on sex, WHO-subtype, FABsubtype, WBC, BPC and cytogenetics $(P>0.05$ for all comparisons) (Table 4).

In multivariate analysis, SRSF2 mutations were found to be independent poor predictors for OS
(HR, 3.307; 95\% CI, 1.137 to $9.614 ; P=0.028$ ) and PFS (HR, 15.431; 95\% CI, 3.041to 78.312; $P=0.001$ ). Likewise, SETBP1 mutations were independent predictors of poor OS (HR, 9.492; 95\% CI, 1.183to 76.128; $P=0.034)$ (Table 4).

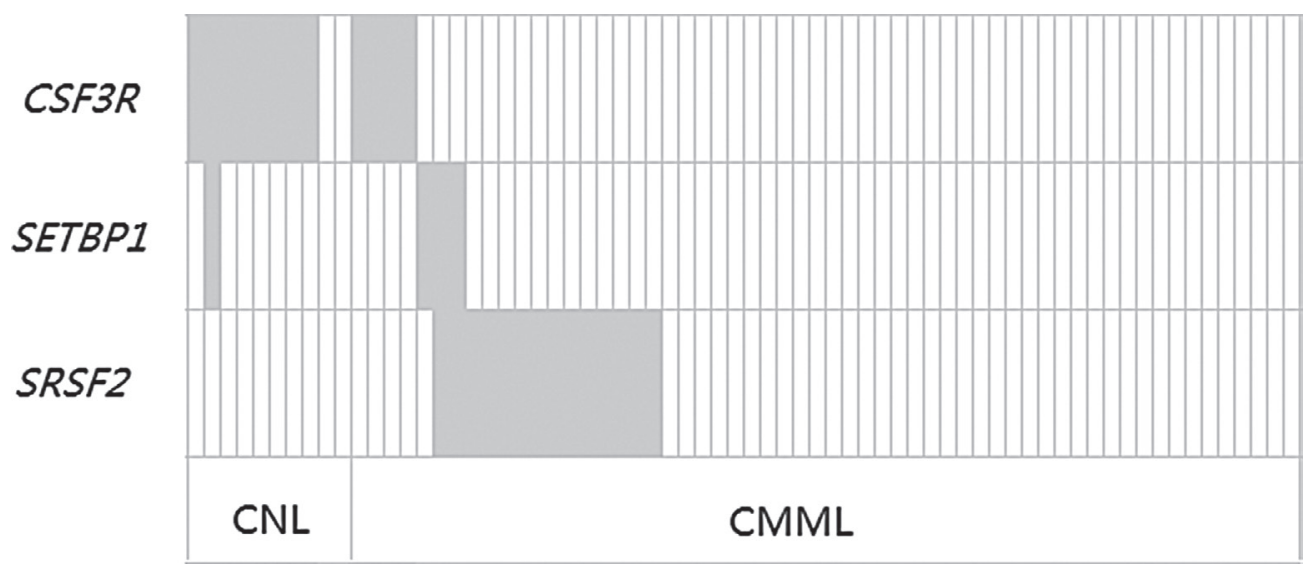

Figure 2: Frequency distribution of $C S F 3 R, S E T B P 1$ and $S R S F 2$ genetic aberrations in CNL and CMML patients. Each box indicates 1 patient. Dark gray boxes are indicative for patients who are positive for the respective mutation; light gray boxes indicate wild type status. 
Table 4: The univariate and multivariate Cox regression analysis of survival in CMML patients

\begin{tabular}{|c|c|c|c|c|c|c|c|c|c|c|c|c|}
\hline \multirow{3}{*}{ Variables } & \multicolumn{6}{|c|}{ OS } & \multicolumn{6}{|c|}{ PFS } \\
\hline & \multicolumn{3}{|c|}{ univariate analysis } & \multicolumn{3}{|c|}{ multivariate analysis } & \multicolumn{3}{|c|}{ univariate analysis } & \multicolumn{3}{|c|}{ multivariate analysis } \\
\hline & $P$ & HR & $95 \% \mathrm{CI}$ & $P$ & HR & $95 \% \mathrm{CI}$ & $P$ & HR & $95 \% \mathrm{CI}$ & $P$ & HR & $95 \% \mathrm{CI}$ \\
\hline SRSF2 mutations & 0.002 & 4.74 & $1.811-12.42$ & 0.028 & 3.31 & $1.14-9.61$ & 0.001 & 15.39 & $3.25-72.81$ & 0.001 & 15.43 & $3.041-78.312$ \\
\hline SETBP1 mutations & 0.002 & 8.25 & $2.12-32.07$ & 0.034 & 9.49 & $1.18-76.13$ & 0.038 & 5.29 & $1.10-25.51$ & NS & NS & NS \\
\hline BM Blast & 0.006 & 1.18 & $1.05-1.32$ & NS & NS & NS & 0.029 & 1.14 & $1.01-1.29$ & NS & NS & NS \\
\hline PB Blast & 0.02 & 1.14 & $1.02-1.27$ & NS & NS & NS & 0.028 & 1.13 & $1.01-1.26$ & NS & NS & NS \\
\hline Mono & 0.015 & 1.13 & $1.02-1.25$ & NS & NS & NS & NS & NS & NS & NS & NS & NS \\
\hline Age & 0.017 & 1.04 & $1.01-1.07$ & NS & NS & NS & NS & NS & NS & NS & NS & NS \\
\hline $\mathrm{HB}$ & NS & NS & NS & NS & NS & NS & 0.038 & 0.941 & $0.941-0.998$ & NS & NS & NS \\
\hline Sex & NS & NS & NS & NS & NS & NS & NS & NS & NS & NS & NS & NS \\
\hline WHO-subtype & NS & NS & NS & NS & NS & NS & NS & NS & NS & NS & NS & NS \\
\hline FAB-subtype & NS & NS & NS & NS & NS & NS & NS & NS & NS & NS & NS & NS \\
\hline WBC & NS & NS & NS & NS & NS & NS & NS & NS & NS & NS & NS & NS \\
\hline $\mathrm{BPC}$ & NS & NS & NS & NS & NS & NS & NS & NS & NS & NS & NS & NS \\
\hline Mono \% & NS & NS & NS & NS & NS & NS & NS & NS & NS & NS & NS & NS \\
\hline Cytogenetics & NS & NS & NS & NS & NS & NS & NS & NS & NS & NS & NS & NS \\
\hline
\end{tabular}

CMML, chronic myelomonocytic leukemia; OS, overall survival; PFS, progression-free survival;BM Blast, blast cell count of bone marrow; PB Blast, blast cell count of peripheral blood; WBC, white blood cell; HB, hemoglobin; BPC, blood platelet cell; Mono, monocyte; HR, hazard ratio; CI, confidence interval.

\section{DISCUSSION}

Next generation sequencing studies have revealed a large number of mutations in genes such as TET2, CBL, AXSL1, RUNX1, EZH2, RAS, JAK2, IDH1/IDH2, NPM1 and spliceosome mutations in CMML [18] as well as CSF3R, SETBP1 mutations in CNL and aCML [19]. In this study, we investigated the frequencies, diagnostic significance and clinical outcome of CSF3R, SETBP1 and $S R S F 2$ gene mutations in CNL and CMML patients.

Our study show that $80 \%$ of CNL patients carried $C S F 3 R$ mutations, with the substitution mutation of $C S F 3 R$ T618I occurring exclusively in WHO-defined CNL. However, CSF3R mutations were not seen in MGUSCNL, MDS, CEL or healthy donors. Pardanani et al. have identified CSF3R T618I in 83\% of CNL patients,
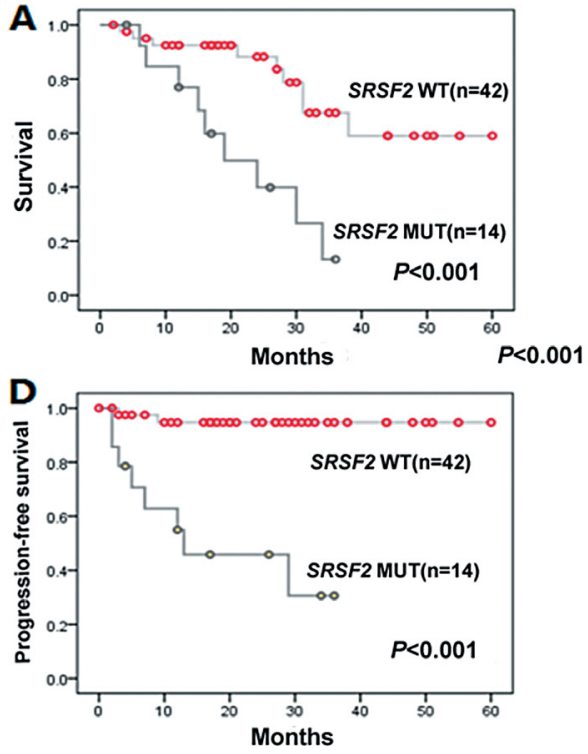
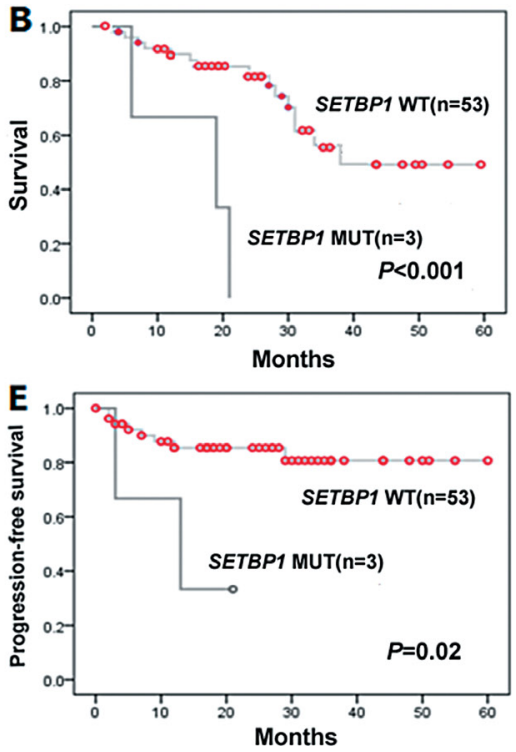
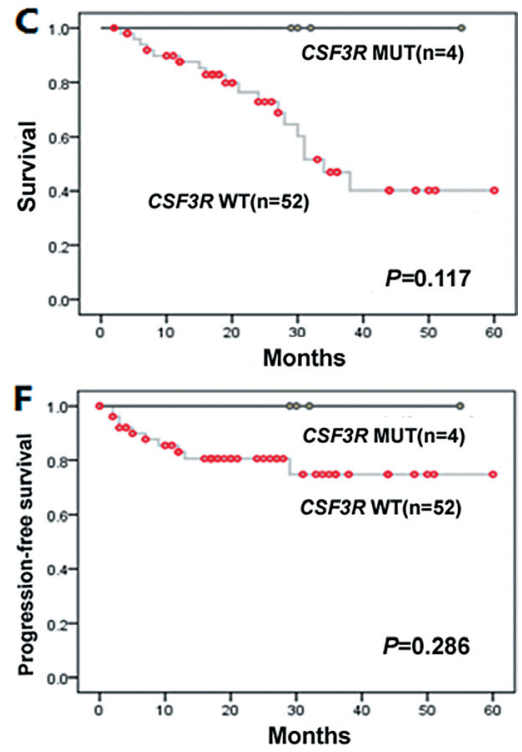

Figure 3: Kaplan-Meier curves for OS, PFS according to genotypes with statistical significance in univariate analysis. (A-C) Overall survival (OS) for $S R S F 2$, SETBP1 and CSF3R cases. (D-F) Progression-free survival (PFS) for $S R S F 2$, SETBP1 and CSF3R cases. In univariate analysis, SRSF2 and SETBP1 mutations suggested a poor prognosis for OS $(P<0.001$ both $)$ and PFS $(P<0.001$ and $P=0.02$, respectively). There was no statistical significance of CSF3R mutations in OS and PFS $(P>0.05)$. 
which did not affect survival [9]. In contrast, patients with SETBP1 mutations (33\% in CNL) experienced shortened survival [9]. Because of the limited number of mutated cases, we only described the clinical characteristics of CNL patients without analyzing the prognostic status of these patients (Table 2).

We identified CSF3R mutations in $7.1 \%$ of CMML patients. Interestingly, all of the $C S F 3 R$ mutations detected in CMML patients were CSF3R P733T mutations. CSF3R P733T mutation represents a novel $C S F 3 R$ point mutation not previously reported. In addition, none of the $4 C S F 3 R$ P733T mutated patients were detected with SRSF2 mutations, suggesting that $C S F 3 R$ P733T mutations and $S R S F 2$ mutations are mutually exclusive in CMML patients. Therefore, the CSF3R P733T mutation may be of diagnostic value in CMML, especially for wt SRSF2 CMML patients. In univariate analysis, we observed that CMML patients with CSF3R mutations had longer PFS and OS compared to wt $C S F 3 R$ patients. However, the difference was not statistically significant. The lack of significance may be due to the small sample size in the $C S F 3 R$ mutant group which resulted in low statistical power. In contrast, Kosmider and collegues identified CSF3R mutations in 3\% CMML patients and showed that $C S F 3 R$ mutations were associated with an reduced OS and AML-free survival in univariate analysis [8]. Additionally, Maxson et al. showed that CSF3R mutations were divided into membrane proximal mutations and truncation mutations based on the distribution within two distinct regions of CSF3R [2]. Truncation mutations in $C S F 3 R$ activates downstream signaling mediators-SRC family kinases (SFKs) and TNK2 and lead to increased sensitivity to Dasatinib treatment [2]. Membrane proximal mutations, on the other hand, showed preferential activation of JAK signaling pathway and susceptible to JAK kinase inhibitors such as Ruxolitinib [2]. Fundamental differences in mutations types might therefore indicate differences in prognosis of CMML patients, which deserves further attention.

Here, we demonstrate SETBP1 mutations present in $5.3 \%$ of CMML patients. In univariate analysis, SETBP1 mutations was an independent adverse predicting factor for OS $(P=0.002)$ and PFS $(P=0.038)$. Similar results were obtained for OS in multivariate analysis. These data are consistent with multiple previous studies [15, 16, 19-21], which have shown that SETBP1 mutations occur in 4-7\% CMML patients and are indicative of decreased OS and AML-free survival [22].

Notably, SRSF2 mutations were detected in 25\% CMML patients in our study but exclusive of mutations in $C S F 3 R$. Our multivariate analysis showed SRSF2 mutation as an independent poor predictor for OS $(P=0.028)$ and PFS $(P=0.001)$, in line with previous data from Itzykson et al. and Makishima et al. [11, 23].

In conclusion, we report that the majority of $\mathrm{CNL}$ patients studied here carried oncogenic CSF3R mutations and that the CSF3R T618I mutation appears to be a diagnostic marker with good specificity and sensitivity for CNL. By contrast, CSF3R P733T mutations detected in CMML patients were completely different from CSF3R mutation types described in patients with CNL, SCN and hereditary neutrophilia, which may be a potential diagnostic marker, particularly for wt SRSF2 CMML patients. In addition, mutations in SRSF2 were commonly found in CMML patients, and represents a poor prognostic marker for CMML. Mutations in SETBP1, by contrast, were found in CNL, CMML but also other hematological malignancies, making it a rather poor isolated prognostic marker for hematological diseases. As patients with different gene mutations may have different clinical response to treatment, mutation-based personalized targeted therapy should be considered in future studies.

\section{MATERIALS AND METHODS}

\section{Study population and definitions}

A total of $10 \mathrm{CNL}$ patients and $56 \mathrm{CMML}$ patients were included in the study. 43 were male and 23 were female, at a median age of 64 (range: 24-94) years. Of the 56 CMML patients, 47 were diagnosed with CMML1 and 9 were diagnosed with CMML-2. Of the $10 \mathrm{CNL}$ patients, 1 was diagnosed with monoclonal gammopathy of undetermined significance associated with chronic neutrophilic leukemia (MGUS-CNL). 20 MDS patients, 10 chronic eosinophilic leukemia (CEL) patients and 20 healthy donors were included as controls. The diagnosis, classification and AML transformation of CMML, CNL, MDS and CEL were based on WHO 2008 criteria [1].

\section{CSF3R, SRSF2 and SETBP1 mutation analysis}

We obtained DNA specimens from bone marrow mononuclear cells or peripheral-blood granulocytes from the patients, and constitutional DNA samples from matched buccal swabs as described previously [9]. $C S F 3 R$ exons 14-17, SETBP1 exon 4 and SRSF2 exon 1 were amplified. Primers used were: $C S F 3 R$ exon $14 \mathrm{~F}$ : CCACGGA GGCAGCTTTAC; CSF3R exon 14 R: AAATCAGCATC CTTTGGG TG. $C S F 3 R$ exon 15 F: TGACTTTGAATCC CCTGGTC; $C S F 3 R$ exon 15 R: TGAGGTTCCCTGT GGGTG; $C S F 3 R$ exon 16 F: AAAATGGAAAGATC GGAG GG; $C S F 3 R$ exon 16 R: CTTGGCTTCAGAAG GTGTCC; CSF3R exon 17 F: CTGTCACTTCCGGC AACAT; $C S F 3 R$ exon 17 R: TGGCCCAAAGACAC AG TCGT; SETBP1 exon $4 \mathrm{~F}$ : ACCTGGAAGCTGTCTCC ACCCA; SETBP1 exon 4 R: CGGTGGCCATGCCGG TTCTT; $S R S F 2$ exon $1 \mathrm{~F}$ : CTGTCACTTCCGGC AACAT; SRSF2 exon $1 \mathrm{R}$ : TGGCCCAAAGA CACAGTCGT.

Independent validations of the detected variants were conducted using Sanger sequencing. If nonsynonymous sequence changes were detected, we 
determined whether the sequence variant had previously been reported as a SNP by searching in the Single Nucleotide Polymorphism database (https://www.ncbi. nlm.nih.gov/projects/SNP/). The previously unreported variants were resequenced with the matched buccal swabs samples DNA from the same patients. The somatic mutation was confirmed when it associated with the bone marrow or peripheral-blood sample DNA and not the matched buccal swabs DNA.

\section{Study of clinical features}

Patient data was collected at the first diagnosis. The median follow-up period was 20 months (range: 2-60). CMML patients were divided into high, intermediate-1, intermediate-2 and low risk group according to the CMML Prognostic Scoring System (CPSS) [24].

\section{Statistical analysis}

The statistical analysis of data was done by using Excel and SPSS (statistical package for social science) version 17.0. Statistical analysis was performed by comparison between groups using Kruskal-Wallis test regarding quantitative nonparametric data and chi-square test regarding qualitative data. The overall survival analysis was done by Kaplan-Meier curve. Multivariate analysis of survival was carried out by Cox regression. All $P$-values $<0.05$ (two-tailed) were considered statistically significant.

\section{ACKNOWLEDGMENTS AND FUNDING}

This work was supported by the National Natural Science Foundation of China (Grant n. 81170490, 81400095), National Public Health Grand Research Foundation (n. 201202017), Project Funded by the Priority Academic Program Development of Jiangsu Higher Education Institute (n. JX10231801), the Program for Development of Innovative Research Teams in the First Affiliated Hospital of Nanjing Medical University, National Science \& Technology Pillar Program (n. 2014BAI09B12), and Project of National Key Clinical Specialty.

\section{CONFLICTS OF INTEREST}

The authors declare no conflicts of interest.

\section{Authors'contributions}

S.-J.Z was the principal investigator who conceived the study; Y.OY. and C.Q. performed most of the experiments; S.-J.Z. participated in the validation experiments; Y.OY. and C.Q. contributed in sample treating, PCR amplification and sequence analysis; Y.OY. and C.Q. gathered detailed clinical information for the study and helped to perform the clinical analysis; and Y.OY. and C.Q. wrote the manuscript.

\section{REFERENCES}

1. Tefferi A, Thiele J, Vannucchi AM, Barbui T. An overview on CALR and CSF3R mutations and a proposal for revision of WHO diagnostic criteria for myeloproliferative neoplasms. Leukemia. 2014; 28:1407-1413.

2. Maxson JE, Gotlib J, Pollyea DA, Fleischman AG, Agarwal A, Eide CA, Bottomly D, Wilmot B, McWeeney SK, Tognon CE, Pond JB, Collins RH, Goueli B, et al. Oncogenic CSF $3 R$ mutations in chronic neutrophilic leukemia and atypical CML. N Engl J Med. 2013; 368:1781-1790.

3. Mehta HM, Glaubach T, Long A, Lu H, Przychodzen B, Makishima H, McDevitt MA, Cross NC, Maciejewski J, Corey SJ. Granulocyte colony-stimulating factor receptor T595I (T618I) mutation confers ligand independence and enhanced signaling. Leukemia. 2013; 27:2407-2410.

4. Touw IP, Beekman R. Severe congenital neutropenia and chronic neutrophilic leukemia: an intriguing molecular connection unveiled by oncogenic mutations in CSF3R. Haematologica. 2013; 98:1490-1492.

5. Aref S, El-Ghonemy M, Abouzeid T, El-Sabbagh A, El-Baiomy M. Prevalence and impact of colony stimulating factor 3 receptor $(C S F 3 R)$ mutations among Egyptian acute myeloid leukemia patients. Leuk Res. 2014; 38:722-725.

6. Beekman R, Valkhof M, van Strien P, Valk PJ, Touw IP. Prevalence of a new auto-activating colony stimulating factor 3 receptor mutation (CSF3R-T595I) in acute myeloid leukemia and severe congenital neutropenia. Haematologica 2013; 98: e62-e63.

7. Cui Y, Li B, Gale RP, Jiang Q, Xu Z, Qin T, Zhang P, Zhang Y, Xiao Z. CSF3R, SETBP1 and CALR mutations in chronic neutrophilic leukemia. J Hematol Oncol. 2014; 15:7-77.

8. Kosmider O, Itzykson R, Chesnais V, Lasho T, Laborde R, Knudson R, Gauthier A, Merlevede J, Ades L, Morabito M, Fontenay M, Tefferi A, Droin N, et al. Mutation of the colony-stimulating factor-3 receptor gene is a rare event with poor prognosis in chronic myelomonocytic leukemia. Leukemia. 2013; 27:1946-1949.

9. Pardanani A, Lasho TL, Laborde RR, Elliott M, Hanson CA, Knudson RA, Ketterling RP, Maxson JE, Tyner JW, Tefferi A. CSF3R T618I is a highly prevalent and specific mutation in chronic neutrophilic leukemia. Leukemia. 2013; 27:1870-1873.

10. Itzykson R, Solary E. An evolutionary perspective on chronic myelomonocytic leukemia. Leukemia. 2013; 27:1441-1450.

11. Itzykson R, Kosmider O, Renneville A, Gelsi-Boyer V, Meggendorfer $\mathrm{M}$, Morabito $\mathrm{M}$, Berthon $\mathrm{C}$, Adès $\mathrm{L}$, Fenaux P, Beyne-Rauzy O, Vey N, Braun T, Haferlach T, et al. Prognostic score including gene mutations in 
chronic myelomonocytic leukemia. J Clin Oncol. 2013; $31: 2428-2436$.

12. Pellagatti A, Boultwood J. The molecular pathogenesis of the myelodysplastic syndromes. Eur J Haematol. 2015; 95:3-15.

13. Chesnais V, Kosmider O, Damm F, Itzykson R, Bernard OA, Solary E, Fontenay M. Spliceosome mutations in myelodysplastic syndromes and chronic myelomonocytic leukemia. Oncotarget. 2012; 3:1284-1293. doi: 10.18632/ oncotarget.749.

14. Senín A, Arenillas L, Martínez-Avilés L, FernándezRodríguez C, Bellosillo B, Florensa L, Besses C, ÁlvarezLarrán A. Molecular characterization of atypical chronic myeloid leukemia and chronic neutrophilic leukemia. Med Clin (Barc). 2015; 144:487-490.

15. Piazza R, Valletta S, Winkelmann N, Redaelli S, Spinelli R, Pirola A, AntoliniL, Mologni L, Donadoni C, Papaemmanuil E, Schnittger S, Kim DW, Boultwood J, et al. Recurrent $S E T B P 1$ mutations in atypical chronic myeloid leukemia. Nat Genet. 2013; 45:18-24.

16. Damm F, Itzykson R, Kosmider O, Droin N, Renneville A, Chesnais V, Gelsi-BoyerV, de Botton S, Vey N, Preudhomme C, Clavert A, Delabesse E, Park S, et al. SETBP1 mutations in 658 patients with myelodysplastic syndromes, chronic myelomonocytic leukemia and secondary acute myeloid leukemias. Leukemia. 2013; 27:1401-1403.

17. Wassie EA, Itzykson R, Lasho TL, Kosmider O, Finke CM, Hanson CA, Ketterling RP, Solary E, Tefferi A, Patnaik MM. Molecular and prognostic correlates of cytogenetic abnormalities in chronic myelomonocytic leukemia: a Mayo Clinic-French Consortium Study. Am J Hematol. 2014; 89:1111-1115.

18. Gotlib J, Maxson JE, George TI, Tyner JW. The new genetics of chronic neutrophilic leukemia and atypical CML: implications for diagnosis and treatment. Blood. 2013; 122:1707-1711.
19. Meggendorfer M, Bacher U, Alpermann T, Haferlach C, Kern W, Gambacorti-Passerini C, Haferlach T, Schnittger S. SETBP1 mutations occur in $9 \%$ of MDS/MPN and in $4 \%$ of MPN cases and are strongly associated with atypical CML, monosomy 7, isochromosome i(17)(q10), ASXL1 and CBL mutations. Leukemia. 2013; 27:1852-1860.

20. Laborde RR, Patnaik MM, Lasho TL, Finke CM, Hanson CA, Knudson RA, Ketterling RP, Pardanani A, Tefferi A. SETBP1 mutations in 415 patients with primary myelofibrosis or chronic myelomonocytic leukemia: independent prognostic impact in CMML. Leukemia. 2013; 27:2100-2102.

21. Thol F, Suchanek KJ, Koenecke C, Stadler M, Platzbecker U, Thiede C, Schroeder T, Kobbe G, Kade S, Löffeld P, Banihosseini S, Bug G, Ottmann O, et al. SETBP1 mutation analysis in 944 patients with MDS and AML. Leukemia. 2013; 27:2072-2075.

22. Cui Y, Tong H, Du X, Li B, Gale RP, Qin T, Liu J, Xu Z, Zhang Y, Huang G, Jin J, Fang L, Zhang H, et al. Impact of TET2, SRSF2, ASXL1 and SETBP1 mutations on survival of patients with chronic myelomonocytic leukemia. Exp Hematol Oncol. 2015; 20:4-14.

23. Makishima H, Visconte V, Sakaguchi H, Jankowska AM, Abu KS, Jerez A, Przychodzen B, Bupathi M, Guinta K, Afable MG, Sekeres MA, Padgett RA, Tiu RV, et al. Mutations in the spliceosome machinery, a novel and ubiquitous pathway in leukemogenesis. Blood. 2012; 119:3203-3210.

24. Such E, Germing U, Malcovati L, Cervera J, Kuendgen A, Della PM, Nomdedeu B, Arenillas L, Luño E, Xicoy B, Amigo ML, Valcarcel D, Nachtkamp K, et al. Development and validation of a prognostic scoring system for patients with chronic myelomonocytic leukemia. Blood. 2013; 121:3005-3015. 